\title{
Effets des différents niveaux de fertilisation aux fientes de poules sur la croissance et la production de biomasse de Brachiaria ruziziensis (Poaceae) en fonction des stades phénologiques à l'Ouest-Cameroun
}

\section{Gilles JIOPE AZANGUE ${ }^{1}$, Victor François NGUETSOP ${ }^{1}$, Fernand TENDONKENG ${ }^{2 *}$, David FOKOM WAUFFO ${ }^{2}$ et Etienne TEDONKENG PAMO ${ }^{2}$}
${ }^{1}$ Laboratoire de Botanique Appliquée, Département de Biologie Végétale, Université de Dschang, Cameroun.
${ }^{2}$ Laboratoire de Nutrition Animale, Département des productions Animales, Université de Dschang, Cameroun.
*Auteur correspondant, E-mail: ftendonkeng@yahoo.fr

\section{RÉSUMÉ}

L'effet des différents niveaux de fertilisation aux fientes de poules sur la croissance et la production de biomasse de B. ruziziensis en fonction des stades phénologiques a été étudié à l'Ouest-Cameroun. Cette étude avait pour objectif d'évaluer les effets des différents niveaux de fertilisation aux fientes de poules sur la croissance et la production de biomasse de $B$. ruziziensis à différents stades phénologiques. Un dispositif factoriel comparant cinq doses d'azote sous forme de fientes de poules $(0 ; 25 ; 50 ; 75$ et $100 \mathrm{~kg} \mathrm{~N} / \mathrm{ha})$ et trois stades phénologiques (montaison, floraison et après grenaison) sur des parcelles de $6 \mathrm{~m}^{2}(3 \mathrm{x} 2 \mathrm{~m})$ en quatre répétitions, soit un total de 60 parcelles expérimentales a été utilisé. Les fientes de poules ont été répandues un mois après la plantation des éclats de souches. À chaque stade phénologique, 40 plantes ont été prélevées par traitement pour les mesures des hauteurs et des diamètres. L'évaluation de la biomasse des tiges, des feuilles et de la plante entière s'est faite sur chaque parcelle en fonction du niveau de fertilisation et du stade phénologique. Les résultats obtenus ont montré que la fertilisation aux fientes de poules a augmenté de manière significative la taille, le diamètre, les biomasses des feuilles, des tiges et des plantes entières des parcelles fertilisées de $B$. ruziziensis par rapport à celui des parcelles témoins et proportionnellement aux quantités de fientes répandues. Les tailles, les diamètres et les biomasses des plantes entières les plus élevées ont été obtenues avec la fertilisation à la dose de $100 \mathrm{~kg} \mathrm{~N} /$ ha. L'étude a montré que les fientes de poules dans les conditions de cet essai ont augmenté la disponibilité en éléments nutritifs du sol et ont pu fournir la quantité d'éléments nutritifs nécessaires à la culture de $B$. ruziziensis.

(C) 2019 International Formulae Group. All rights reserved

Mots clés : Brachiaria ruziziensis, fiente de poules, taille, diamètre, biomasse, Dschang.

\section{Effects of different levels of fertilization with chicken manure on the growth and biomass production of Brachiaria ruziziensis (Poaceae) according to phenological stages in West Cameroon}

\begin{abstract}
The effect of different levels of chicken manure fertilization on the growth and biomass production of B. ruziziensis according to phenological stages was studied in West Cameroon. The objective of this study was to evaluate the effects of different levels of chicken manure fertilization on the growth and biomass production
\end{abstract}


of B. ruziziensis at different phenological stages. A factorial device comparing five doses of nitrogen in the form of chicken manure $(0 ; 25 ; 25 ; 50 ; 75$ and $100 \mathrm{~kg} \mathrm{~N} / \mathrm{ha}$ ) and three phenological stages (bolting, flowering and after seed set) on $6 \mathrm{~m}^{2}(3 \times 2 \mathrm{~m})$ plots in four replicates, for a total of 60 experimental plots was used. The chicken manure were spread one month after the planting of the stump chips. At each phenological stage, 40 plants were collected per treatment for height and diameter measurements. The evaluation of stem, leaf and whole plant biomass was carried out on each plot according to the level of fertilization and phenological stage. The results obtained showed that fertilization with chicken manure significantly increased the height, diameter, biomass of leaves, stems and whole plants of the fertilized plots of B. ruziziensis compared to that of the control plots and in proportion to the quantities of chicken manure spread. The highest plants, diameters and biomass of the highest whole plants were obtained with fertilization at $100 \mathrm{~kg} \mathrm{~N} / \mathrm{ha}$. The study showed that chicken manure under the conditions of this test increased the availability of soil nutrients and were able to provide the amount of nutrients needed to grow $B$. ruziziensis.

(C) 2019 International Formulae Group. All rights reserved

Keywords: Brachiaria ruziziensis, chicken manure, height, diameter, biomass, Dschang.

\section{INTRODUCTION}

La nécessité de nourrir une population sans cesse croissante dans les pays en développement pousse les agriculteurs à l'exploitation des terres marginales longtemps considérées comme impropres à l'agriculture (Tendonkeng et al., 2010), à étendre les surfaces cultivables au détriment des espaces pastoraux, ce qui a pour conséquence le développement de conflits pour l'utilisation de l'espace rural entre agriculteurs et éleveurs (Pamo et al., 2006 ; Tendonkeng et al., 2011 ; Sawa et al., 2018). Cette situation conduit au rétrécissement des parcours naturels (Pamo et al., 2007; Tendonkeng et al., 2011a; Tendonkeng et al., 2011b; Muhindo et al., 2018 ; Sawa et al., 2018), à leur déplacement vers les zones marginales (Pamo et al., 2008) déjà surexploitées. Les prairies naturelles, qui ont été jusqu'ici utilisées de façon extensive, s'épuisent et perdent leur capacité d'élevage du bétail (JGRC, 2001). Il devient donc nécessaire de développer un élevage de type sédentaire qui, étant fondé sur le travail actif des terres, en assure la conservation et en élève la productivité (JGRC, 2001). Ce type d'élevage permet en effet une utilisation intensive et durable des ressources terrestres, et facilite la gestion du bétail. Et si l'on vise le passage de l'élevage de type nomade à l'élevage de type sédentaire, il apparaît alors la nécessité de faire augmenter l'approvisionnement en fourrage à l'intérieur de la zone sédentaire (JGRC, 2001). La culture fourragère peut constituer une alternative dans les pays tropicaux. Elle permettrait ainsi d'assurer une disponibilité tant en période favorable (saison des pluies) qu'en période défavorable (saison sèche) (Obulbiga et Kaboré-Zoungrana, 2007, Tendonkeng, 2011). Dans ce cas les systèmes de productions n'auront plus comme seul objectif de produire du lait et de la viande, mais permettront également de produire un fourrage de bonne qualité avec des techniques culturales accessibles aux paysans (Mboko et al., 2013).

Parmi les nombreuses espèces fourragères introduites au Cameroun, figure Brachiaria ruziziensis. Cette graminée pérenne originaire d'Afrique centrale (Rwanda, Burundi et surtout de la plaine de Ruzizi en République Démocratique du Congo) présente les avantages de mieux s'adapter dans différentes régions, d'avoir une bonne valeur nutritive et d'être bien appétée par les animaux (Pamo et al., 2007; Tendonkeng et al., 2011). Elle est proposée pour être utilisé avec profit non seulement dans l'amélioration des pâturages naturels, mais également comme un fourrage vert ou conservé (Cook et al., 2005 ; Tendonkeng et al., 2011). La culture de B. ruziziensis et surtout l'intensification de son utilisation peuvent ainsi constituer un palliatif au rétrécissement des parcours naturels (Tendonkeng, 2011). Elle permettrait d'améliorer les rations des animaux tout au 
long de l'année. Les plantes du genre Brachiaria sont capables de puiser le peu d'éléments nutritifs qui restent dans des sols pauvres. En conséquence, leur exploitation intensive comme fourrages, sans apport de fertilisation (engrais ou fumier) en compensation risque de conduire à un épuisement des sols qui deviendront incultes (Husson et al., 2008). Prenant en compte le coût élevé des engrais minéraux et leur rôle néfaste dans l'écologie des sols, l'utilisation de la fiente de poule représente un potentiel énorme de gestion de la fertilité des sols et d'amélioration de la croissance et des rendements des cultures (Yerima et al., 2014). C'est dans cette optique que s'inscrit cette étude qui a pour objectif d'évaluer l'effet des fientes de poules sur la croissance et la production de biomasse de B. ruziziensis. Il s'agit de déterminer le niveau optimal de certains éléments nutritifs tel que l'azote contenu dans les fientes de poules et ses effets immédiats ou lointains sur la croissance et la production de biomasse de la plante dans le temps.

\section{MATERIEL ET METHODES Zone d'étude}

L'étude a été conduite à la Ferme d'Application et de Recherche (FAR) de l'Université de Dschang entre avril et décembre 2015. La FAR est située dans la région de l'Ouest Cameroun, dans le département de la Menoua, arrondissement de Dschang, à $05^{\circ} 20^{\prime}$ latitude Nord et $10^{\circ} 03^{\prime}$ longitude Est. Le climat de toute l'étendue du territoire est un climat équatorial camerounien à faciès montagnard déterminé par l'altitude dont la moyenne est de $1400 \mathrm{~m}$. Il est caractérisé par une saison des pluies allant de mi-mars à mi-novembre et une saison sèche s'étendant de mi-novembre à mi-mars. La température moyenne du mois le plus chaud (février) est de $25{ }^{\circ} \mathrm{C}$ et celle du mois le plus frais est de $10{ }^{\circ} \mathrm{C}$ et se situe en juillet ou en août. L'insolation annuelle est de 1800 heures, l'humidité relative variant entre 40 et $97 \%$. Les précipitations varient entre 1500 et 2000 mm par an (Pamo et al., 2005 ; Tendonkeng, 2011).

\section{Dispositif expérimental}

Un dispositif factoriel $\left(\begin{array}{lll}5 & \mathrm{x} & 3\end{array}\right)$ comparant cinq doses d'azote sous forme de fientes de poules $(0 ; 25 ; 50 ; 75 ; 100 \mathrm{~kg}$ $\mathrm{N} / \mathrm{ha}$ ) et trois stades phénologiques de coupe (montaison, floraison et après grenaison) sur des parcelles de $6 \mathrm{~m}^{2}$ ( $\left.3 \times 2 \mathrm{~m}\right)$ en quatre répétitions, soit un total de 60 parcelles expérimentales a été utilisé.

\section{Origine et composition chimique du fertilisant}

Le fertilisant organique utilisé au cours de cette étude est la fiente de poule. Elle a été obtenue d'un élevage chez un producteur dans le département de la Menoua (Dschang). Sa composition chimique a été analysée au Laboratoire d'Analyse des Sols et de Chimie de l'Environnement (LABASCE) de l'Université de Dschang dans le but de déterminer les quantités à appliquer. Les résultats de l'analyse de la composition chimique de la fiente de poule ont été consignés dans le Tableau 1.

\section{Préparation du sol, mise en place des plants et fertilisation}

Les échantillons du sol ont été prélevés sur le site de l'essai dans l'horizon 0 - $20 \mathrm{~cm}$ de profondeur avant la préparation du sol et la mise en place des éclats de souche. L'analyse chimique du sol a été effectuée au LABASCE. Les résultats ont montré que la classe texturale moyenne de ce sol est limoneuse. Ce sol est légèrement acide avec un $\mathrm{pH}$ moyen de 5,5 , ce qui exclut le risque de toxicité due à un excès d'aluminium et de manganèse. Les teneurs en azote total sont faibles $(0,6 \mathrm{~g} / \mathrm{kg}$ de sol). Pour la plupart des sols camerounais, des teneurs de $1 \mathrm{~g} / \mathrm{kg}$ permettent déjà de conduire un cycle de culture en agriculture traditionnelle, mais pour une culture intensive, ces teneurs doivent être augmentées à $2 \mathrm{~g} / \mathrm{kg}$. Il faut donc prévoir un complément azoté. Le taux de matière organique (avec un ratio $\mathrm{C} / \mathrm{N}=11,66$ ) est compris dans la fourchette idéale $(8-12 \%)$ traduisant une bonne minéralisation de la matière organique. Ceci confère à cette terre une capacité de rétention plus grande pouvant lui permettre de supporter une fertilisation 
massive. Pour ce qui est des bases échangeables, le sol est faiblement pourvu en magnésium, en potassium, en calcium et en phosphore. Le complexe absorbant de ce sol présente une capacité d'échange cationique élevée $(7,84 \mathrm{cmol} / \mathrm{kg}$ de sol $)$ du fait de la matière organique élevé. Ces caractéristiques permettent d'envisager sans crainte une fertilisation avec des doses élevées d'engrais. En effet, le niveau de fertilité d'un sol est optimal lorsque les conditions physicochimiques et climatiques propres à un cultivar donné sont pourvus (Tendonkeng, 2011). Pour le cas du B. ruziziensis qui exige un sol d'une fertilité élevée, ces conditions sont acceptables dans les hautes terres de l'Ouest Cameroun et les apports subséquents pourront modifier favorablement cet environnement dans le but d'améliorer sa culture.

La préparation des planches de semis de $6 \mathrm{~m}^{2}(3 \times 2 \mathrm{~m})$ a été faite manuellement à l'aide d'un décamètre et des piquets qui ont servi à délimiter les planches et les couloirs ; les machettes, les houes et les pioches ont servi à l'élaboration des planches et l'ameublissement du sol. Des éclats de souches comportant plusieurs plants de $B$. ruziziensis ont été prélevés dans le parcours de la FAR, puis habillées (par réduction de la taille des feuilles et des racines) à l'aide d'une machette et repiquées sur chacune des parcelles à $4 \mathrm{~cm}$ de profondeur, suivant un écartement de $20 \mathrm{~cm}$ x $15 \mathrm{~cm}$ respectivement entre et sur la ligne.

Les niveaux de fertilisation à la fiente de poules ont été définis en terme de quantité d'azote contenue dans la fiente de poule non seulement parce que l'azote est l'élément nutritif le plus déficient dans les systèmes de production agricoles à travers son rôle dans la synthèse de la matière vivante (Mihoub, 2008), mais aussi parce que c'est un facteur de rendement et parfois de qualité qui augmente la teneur des protéines dans les plantes (Soltner, 2003). Ainsi, cinq doses d'azote ont été définis $(0,25,50,75$ et $100 \mathrm{~kg}$ d'azote à l'hectare) pour la culture de B. ruziziensis. À chacune des doses correspondait une quantité précise de fientes de poules telle que résumée dans le Tableau 2. Un mois après la plantation des éclats de souche, les parcelles ont été fertilisées à l'aide de la fiente de poule une seule fois suivant les quantités indiquées dans le Tableau 2. L'entretien des parcelles a consisté chaque mois, au désherbage manuel des adventices.

\section{Collecte des données}

\section{Évaluation de la croissance}

À chacun des trois stades phénologiques, dix (10) plantes étaient prélevées de manière aléatoire dans chaque répétition, pour un total de 40 plantes par traitement pour évaluer les mensurations (hauteurs et diamètres). Les hauteurs des plantes (du point de fauche à l'extrémité de la plus longue feuille) ont été mesurées à l'aide d'un mètre ruban gradué au centimètre et les diamètres ont été mesurés (avant le premier entre-nœud) à l'aide d'un pied à coulisse électronique graduée au millimètre.

\section{Évaluation de la biomasse}

À chaque stade phénologique, 20 planches (soit quatre répétitions par traitement) ont été fauchées à l'aide d'une table de coupe et des couteaux bien tranchants. Pendant la fauche et pour éviter les effets de bordure, B. ruziziensis a été fauché à $5 \mathrm{~cm}$ du sol sur une surface utile de $2 \mathrm{~m}^{2}(2 \mathrm{x}$ $1 \mathrm{~m})$ au centre de la planche. Le fourrage ainsi récolté a été ensuite pesé. Un échantillon représentatif de $0,5 \mathrm{~kg}$ de $B$. ruziziensis a été prélevé à chaque stade phénologique par niveau de fertilisation sur chaque bloc. Chaque échantillon a été ensuite séparé en feuilles et en tiges qui ont été découpés et séché à $60{ }^{\circ} \mathrm{C}$ dans une étuve ventilée de Marque Gallemkamp jusqu'à poids constant pour la détermination de la matière sèche et l'évaluation du rendement.

\section{Analyse statistique}

Les données sur la hauteur, le diamètre et la biomasse produite ont été soumises à une analyse de variance multifactorielle suivant le Modèle Linéaire Général (MLG) à l'aide du logiciel SPSS version 20.0. Lorsqu'il existait des différences entre les différents traitements, les moyennes étaient séparées par le test de Duncan au seuil de signification $5 \%$. 
Tableau 1 : Composition chimique de la fiente de poule.

\begin{tabular}{lllllll}
\hline & N & K & P & Na & Ca & Mg \\
\hline $\begin{array}{l}\text { Teneur (en } \\
\mathrm{mg} / \mathrm{kg})\end{array}$ & 2600 & 20438 & 809 & 1015 & 6960 & 73046 \\
\hline
\end{tabular}

Tableau 2 : Quantités d'engrais organique appliqués.

\begin{tabular}{ccc}
\hline $\begin{array}{c}\text { Niveaux de } \\
\text { fertilisation }\end{array}$ & $\begin{array}{c}\text { Quantité de fiente appliquée } \\
\text { (kg / parcelle) }\end{array}$ & $\begin{array}{c}\text { Dose d'azote équivalente } \\
(\mathbf{k g ~ N} / \mathbf{h a})\end{array}$ \\
\hline $\mathbf{T}_{\mathbf{0}}$ & 0 & 0 \\
\hline $\mathbf{T}_{\mathbf{1}}$ & 5,77 & 25 \\
\hline $\mathbf{T}_{\mathbf{2}}$ & 11,53 & 50 \\
\hline $\mathbf{T}_{\mathbf{3}}$ & 17,30 & 75 \\
\hline $\mathbf{T}_{\mathbf{4}}$ & 23,07 & 100 \\
\hline
\end{tabular}

\section{RÉSULTATS}

Effets des différents niveaux de fertilisation à la fiente de poules sur la taille de Brachiaria ruziziensis à différents stades phénologiques

La variation de la taille de $B$. ruziziensis sous l'effet des niveaux croissants de fertilisation à la montaison, floraison et après grenaison est présentée dans le Tableau 3.

Il ressort du Tableau 3 que la taille de B. ruziziensis a augmenté avec la fertilisation jusqu'à la dose de $100 \mathrm{~kg} \mathrm{~N} / \mathrm{ha}$ à la montaison, floraison et après grenaison. À la montaison, les tailles des plantes fertilisées aux doses de 50,75 et $100 \mathrm{~kg} \mathrm{~N} / \mathrm{ha}$ (respectivement 160,42;163,82 et $167,70 \mathrm{~cm}$ ) ont été supérieures $(\mathrm{p}<0,05)$ à celle des plantes des parcelles non fertilisées. Par contre, la taille des plantes des parcelles fertilisées à la dose de $25 \mathrm{~kg} \mathrm{~N} / \mathrm{ha}(157,47 \mathrm{~cm})$ a été statistiquement comparable $(\mathrm{p}>0,05)$ à la taille des plantes des parcelles témoins $(153,42 \mathrm{~cm})$. À la floraison, la taille des plantes des parcelles fertilisées a été supérieure $(\mathrm{p}<0,05)$ à celle des parcelles témoins. Les tailles des plantes ont augmentées de manière significative $(p<0,05)$ avec les niveaux croissants de fertilisation aux fientes de poules jusqu'à la dose de $100 \mathrm{~kg}$ $\mathrm{N} / \mathrm{h}$. Après grenaison, les tailles des plantes des parcelles fertilisées ont été supérieures $(\mathrm{p}<0,05)$ à celles des parcelles non fertilisées. Les tailles des plantes des parcelles fertilisées aux doses de 25,50 et $75 \mathrm{~kg} \mathrm{~N} / \mathrm{ha}$ (respectivement 218,$70 ; 219,47$; et 220,67 $\mathrm{cm})$, ont été comparables et inférieures $(\mathrm{p}<0,05)$ à celles des parcelles fertilisées à la dose de $100 \mathrm{~kg} \mathrm{~N} / \mathrm{ha}(223,65 \mathrm{~cm})$.

Effets des différents niveaux de fertilisation à la fiente de poules sur le diamètre de Brachiaria ruziziensis à différents stades phénologiques

La variation du diamètre de $B$. ruziziensis en fonction des différents niveaux de fertilisation à différents stades phénologiques est présentée dans le Tableau 4.

Quel que soit le stade phénologique, la fertilisation a affecté $(\mathrm{p}<0,05)$ le diamètre de B. ruziziensis. En effet, à la montaison, la fertilisation a permis d'obtenir des plantes avec un diamètre plus grand $(p<0,05)$ que celui des parcelles non fertilisées. Ainsi, les diamètres des plantes des parcelles fertilisées 
aux doses de $25,50,75$ et $100 \mathrm{~kg} \mathrm{~N} / \mathrm{ha}$ (respectivement 4,$02 ; 4,10 ; 4,00$ et $4,07 \mathrm{~mm}$ ) ont été comparables et plus grands $(p<0,05)$ que celui des plantes des parcelles non fertilisées $(3,60 \mathrm{~mm})$. À la floraison, les diamètres des plantes des parcelles fertilisées ont été plus élevés $(p<0,05)$ que ceux des plantes des parcelles non fertilisées. Les diamètres des plantes des parcelles fertilisées aux doses de 25 et $50 \mathrm{~kg} \mathrm{~N} / \mathrm{ha}$ (respectivement $3,75$ et $3,85 \mathrm{~mm})$ ont été statistiquement comparables $(p>0,05)$. La même tendance a été observée pour les diamètres des plantes des parcelles fertilisées aux doses de 50 et 75 $\mathrm{kg} \mathrm{N} / \mathrm{ha}$ (respectivement 3,85 et $4,00 \mathrm{~mm}$ ) et pour les diamètres des plantes des parcelles fertilisées aux doses de 75 et $100 \mathrm{~kg} \mathrm{~N} / \mathrm{ha}$ (respectivement 4,00 et $4,07 \mathrm{~mm}$ ). Après grenaison, les diamètres des plantes des parcelles non fertilisées $(3,67 \mathrm{~mm})$ et celui des parcelles fertilisées aux doses de $25 \mathrm{~kg} \mathrm{~N} / \mathrm{ha}$ $(3,95 \mathrm{~mm})$ ont été comparables $(\mathrm{p}>0,05)$ mais inférieures $(\mathrm{p}<0,05)$ à celui des parcelles fertilisées aux doses de 50,75 et $100 \mathrm{~kg} \mathrm{~N} / \mathrm{ha}$ (respectivement 4,$10 ; 4,00$ et $4,15 \mathrm{~mm}$ ) dont les diamètres ont été comparables $(p>0,05)$ entre eux.

\section{Effets des différents niveaux de fertilisation} à la fiente de poule sur la production de biomasse de $B$. ruziziensis à la montaison

L'évolution de la biomasse des feuilles, des tiges et de la plante entière de $B$. ruziziensis à la montaison est présentée dans le Tableau 5.

Il ressort de ce tableau que la biomasse des feuilles des parcelles non fertilisées $(8,11 \mathrm{t}$ $\mathrm{MS} / \mathrm{ha})$ a été comparable $(\mathrm{p}>0,05)$ à celles obtenues sur les parcelles fertilisées aux doses de 25, 50, 75 et $100 \mathrm{~kg} \mathrm{~N} / \mathrm{ha}$. Au niveau des tiges et de la plante entière, la fertilisation a permis d'obtenir des biomasses supérieures $(\mathrm{p}<0,05)$ à celles des parcelles non fertilisées. Les biomasses des tiges des parcelles fertilisées aux doses de 50 et $75 \mathrm{~kg} \mathrm{~N} / \mathrm{ha}$ (respectivement 13,17 et 14,42 t MS/ha) ont été comparables et supérieures $(\mathrm{p}<0,05)$ à celle des parcelles fertilisées aux doses de 25 $\mathrm{kg}$ N/ha $(11,27$ t MS/ha). La même observation a été faite entre les biomasses des parcelles fertilisées aux doses de 75 et $100 \mathrm{~kg}$ $\mathrm{N} / \mathrm{ha}$. Les biomasses des plantes entières des parcelles fertilisées ont été supérieures à celles des parcelles témoins $(18,22$ t MS/ha). Les biomasses des plantes entières des parcelles fertilisées aux doses de 50 et $75 \mathrm{~kg} \mathrm{~N} / \mathrm{ha}$ (respectivement 22,37 et 23,11 t MS/ha) ont été comparables et supérieures $(\mathrm{p}<0,05)$ à la biomasse des plantes entières des parcelles fertilisées aux doses de $25 \mathrm{~kg}$ N/ha. De même, les biomasses des plantes entières des parcelles fertilisées aux doses de 75 et $100 \mathrm{~kg}$ $\mathrm{N} / \mathrm{ha}$ (respectivement 23,11 et 24,12 t MS/ha) ont été comparables entre elles.

Effets des différents niveaux de fertilisation à la fiente de poule sur la production de biomasse de $B$. ruziziensis à la floraison

L'évolution de la biomasse des feuilles, des tiges et de la plante entière de $B$. ruziziensis sous l'effet des niveaux croissants de fertilisation à la fiente de poules à la floraison est présentée dans le Tableau 6.

La biomasse des feuilles, des tiges et de la plante entière a variée de manière significative $(p<0,05)$ en fonction des différents niveaux de fertilisation. En effet, les biomasses des feuilles des parcelles fertilisées aux doses de 25,50 et $75 \mathrm{~kg} \mathrm{~N} / \mathrm{ha}$ ont été comparables entre elles et supérieures $(p<0,05)$ à la biomasse des feuilles des parcelles non fertilisées. La biomasse des feuilles la plus élevée a été obtenue sur les parcelles fertilisées aux doses de $100 \mathrm{~kg}$ N/ha (10,63 t MS/ha). La biomasse des tiges des plantes des parcelles non fertilisées $(16,21 \mathrm{t}$ $\mathrm{MS} / \mathrm{ha}$ ) et celle des plantes des parcelles fertilisées à la dose de $25 \mathrm{~kg} \mathrm{~N} / \mathrm{ha}(16,73 \mathrm{t}$ $\mathrm{MS} / \mathrm{ha})$ ont été comparables $(\mathrm{p}>0,05)$ et inférieures à la biomasse de chacune des parcelles fertilisées aux doses de 50, 75 et 100 $\mathrm{kg} \mathrm{N} / \mathrm{ha}$. Par contre, La biomasse des tiges des parcelles fertilisées à la dose de $100 \mathrm{~kg} \mathrm{~N} / \mathrm{ha}$ $(19,44 \mathrm{t}$ MS/ha) a été supérieure $(\mathrm{p}<0,05)$ à celle des parcelles fertilisées à la dose $50 \mathrm{~kg}$ $\mathrm{N} / \mathrm{ha}(17,77 \mathrm{t} \mathrm{MS} / \mathrm{ha})$. La biomasse des tiges la plus élevée au cours de ce stade phénologique a été obtenue avec la fertilisation à la dose de $75 \mathrm{~kg} \mathrm{~N} / \mathrm{ha}(20,88 \mathrm{t}$ $\mathrm{MS} / \mathrm{ha}$ ). L'évolution de la biomasse de la 
plante entière à la floraison a montré que la fertilisation a permis d'obtenir des biomasses supérieures à la biomasse des plantes des parcelles non fertilisées. En effet, les biomasses des plantes entières des parcelles fertilisées aux doses de 25 et $50 \mathrm{~kg} \mathrm{~N} / \mathrm{ha}$ (respectivement 26,04 et 27,12 t MS/ha) ont été comparables entre elles et supérieures $(p<0,05)$ à la biomasse des plantes des parcelles non fertilisées (22,30 t MS/ha). La même tendance a été observée pour les biomasses des plantes entières des parcelles fertilisées aux doses de 75 et $100 \mathrm{~kg} \mathrm{~N} / \mathrm{ha}$.

\section{Effets des différents niveaux de fertilisation} à la fiente de poules sur la production de biomasse de $B$. ruziziensis après grenaison

Le Tableau 7 présente les variations des biomasses des feuilles, des tiges et de la plante entière de $B$. ruziziensis en fonction des différents niveaux de fertilisation après grenaison.

Il découle de ce tableau que les biomasses des feuilles, des tiges et de la plante entière obtenues sur les parcelles fertilisées ont été supérieures $(\mathrm{p}<0,05)$ par rapport à celles des plantes des parcelles non fertilisées. En effet, les biomasses des feuilles des parcelles fertilisées aux doses de 50,75 et 100 $\mathrm{kg} \mathrm{N} / \mathrm{ha}$ (respectivement 7,$13 ; 7,24$ et $7,74 \mathrm{t}$ $\mathrm{MS} / \mathrm{ha})$ ont été comparables $(\mathrm{p}>0,05)$ entre elles, et supérieures $(p<0,05)$ à la biomasse des feuilles des parcelles fertilisées à la dose de $25 \mathrm{~kg} \mathrm{~N} / \mathrm{ha}$. Les biomasses des tiges des parcelles fertilisées aux doses de 25 et $50 \mathrm{~kg}$ $\mathrm{N} /$ ha (respectivement 18,43 et 19,36 t MS/ha) ont été comparables et inférieures $(\mathrm{p}<0,05)$ aux biomasses des tiges des parcelles fertilisées aux doses de 75 et $100 \mathrm{~kg} \mathrm{~N} / \mathrm{ha}$ (respectivement 19,76 et 19,80 t MS/ha) qui ont été comparables $(\mathrm{p}>0,05)$ entre elles. Les biomasses des plantes entières obtenues sur les parcelles fertilisées à la dose de 50 et 75 $\mathrm{kg} \mathrm{N} / \mathrm{ha}$ ont été comparables $(\mathrm{p}>0,05)$ et supérieures $(p<0,05)$ à la biomasse des plantes entières provenant des parcelles fertilisées à la dose de $25 \mathrm{~kg} \mathrm{~N} / \mathrm{ha}$. La même observation a été faite avec les biomasses des plantes entières des parcelles fertilisées à la dose de 75 et $100 \mathrm{~kg} \mathrm{~N} / \mathrm{ha}$.

Tableau 3 : Effets des différents niveaux de fertilisation à la fiente de poule sur la taille (en $\mathrm{cm}) \mathrm{de}$ B. ruziziensis aux différents stades phénologiques.

\begin{tabular}{cccc}
\hline $\begin{array}{c}\text { Niveaux de } \\
\text { fertilisation }\end{array}$ & Montaison & Floraison & Après grenaison \\
\hline $\mathbf{T}_{\mathbf{0}}$ & $153,42^{\mathrm{a}}$ & $207,62^{\mathrm{a}}$ & $209,87^{\mathrm{a}}$ \\
\hline $\mathbf{T}_{\mathbf{1}}$ & $157,47^{\mathrm{ab}}$ & $214,70^{\mathrm{b}}$ & $218,70^{\mathrm{b}}$ \\
\hline $\mathbf{T}_{\mathbf{2}}$ & $160,42^{\mathrm{bc}}$ & $217,20^{\mathrm{c}}$ & $219,47^{\mathrm{b}}$ \\
\hline $\mathbf{T}_{\mathbf{3}}$ & $163,82^{\mathrm{cd}}$ & $218,82^{\mathrm{d}}$ & $220,67^{\mathrm{b}}$ \\
\hline $\mathbf{T}_{\mathbf{4}}$ & $167,70^{\mathrm{d}}$ & $222,75^{\mathrm{e}}$ & 0,49 \\
\hline $\mathbf{E S M}$ & 0,77 & 0,42 & 0,0001 \\
\hline $\mathbf{p}$ & 0,0001 & 0,0001 & \\
\hline
\end{tabular}

a,b,c,d,e : les moyennes portant les mêmes lettres dans la même colonne ne sont pas significatives au seuil de 5\%. ESM : erreur standard des moyennes.

$\mathrm{T}_{0}=$ témoin, $\mathrm{T}_{1}=25 \mathrm{~kg} \mathrm{~N} / \mathrm{ha}, \mathrm{T}_{2}=50 \mathrm{~kg} \mathrm{~N} / \mathrm{ha}, \mathrm{T}_{3}=75 \mathrm{~kg} \mathrm{~N} / \mathrm{ha}, \mathrm{T}_{4}=100 \mathrm{~kg} \mathrm{~N} / \mathrm{ha}$. 
Tableau 4 : Effets des différents niveaux de fertilisation à la fiente de poule sur le diamètre (en $\mathrm{mm}$ ) de $B$. ruziziensis à différents stades phénologiques.

\begin{tabular}{cccc}
\hline $\begin{array}{c}\text { Niveaux de } \\
\text { fertilisation }\end{array}$ & Montaison & Floraison & Après grenaison \\
\hline $\mathbf{T}_{\mathbf{0}}$ & $3,60^{\mathrm{a}}$ & $3,42^{\mathrm{a}}$ & $3,67^{\mathrm{a}}$ \\
\hline $\mathbf{T}_{\mathbf{1}}$ & $4,02^{\mathrm{b}}$ & $3,75^{\mathrm{b}}$ & $3,95^{\mathrm{ab}}$ \\
\hline $\mathbf{T}_{\mathbf{2}}$ & $4,10^{\mathrm{b}}$ & $3,85^{\mathrm{bc}}$ & $4,10^{\mathrm{b}}$ \\
\hline $\mathbf{T}_{\mathbf{3}}$ & $4,00^{\mathrm{b}}$ & $4,00^{\text {cd }}$ & $4,00^{\mathrm{b}}$ \\
\hline $\mathbf{T}_{\mathbf{4}}$ & $4,07^{\mathrm{b}}$ & $4,07^{\mathrm{d}}$ & 0,04 \\
\hline $\mathbf{E S M}$ & 0,04 & 0,03 & 0,012 \\
\hline $\mathbf{p}$ & 0,002 & 0,0001 & \\
\hline $\begin{array}{l}\text { a.b,c,d }: \text { les moyennes portant les mêmes lettres dans la même colonne ne sont pas significatives au seuil de } 5 \% . \\
\mathrm{T}_{0}=\text { témoin, } \mathrm{T}_{1}=25 \mathrm{~kg} \mathrm{~N} / \mathrm{ha}, \mathrm{T}_{2}=50 \mathrm{~kg} \mathrm{~N} / \mathrm{ha}, \mathrm{T}_{3}=75 \mathrm{~kg} \mathrm{~N} / \mathrm{ha}, \mathrm{T}_{4}=100 \mathrm{~kg} \mathrm{~N} / \mathrm{ha} .\end{array}$
\end{tabular}

Tableau 5 : Effets des niveaux de fertilisation à la fiente de poule sur la production de biomasse (en t MS/ha) des feuilles, des tiges et de la plante entière de $B$. ruziziensis à la montaison.

\begin{tabular}{|c|c|c|c|}
\hline $\begin{array}{l}\text { Niveaux de } \\
\text { fertilisation }\end{array}$ & Feuilles & Tiges & Plante entière \\
\hline $\mathbf{T}_{\mathbf{0}}$ & $8,11^{\mathrm{a}}$ & $10,13^{\mathrm{a}}$ & $18,22^{\mathrm{a}}$ \\
\hline$T_{1}$ & $8,65^{\mathrm{a}}$ & $11,72^{b}$ & $20,37^{b}$ \\
\hline $\mathbf{T}_{2}$ & $9,20^{\mathrm{a}}$ & $13,17^{\mathrm{c}}$ & $22,37^{\mathrm{c}}$ \\
\hline $\mathbf{T}_{3}$ & $8,69^{\mathrm{a}}$ & $14,42^{\text {cd }}$ & $23,11^{\text {cd }}$ \\
\hline $\mathbf{T}_{4}$ & $9,09^{\mathrm{a}}$ & $15,03^{\mathrm{d}}$ & $24,12^{\mathrm{d}}$ \\
\hline ESM & 0,14 & 0,44 & 0,50 \\
\hline $\mathbf{p}$ & 0,107 & 0,0001 & 0,0001 \\
\hline
\end{tabular}


Tableau 6 : Effets des niveaux de fertilisation à la fiente de poules sur la production de biomasse (en t MS/ha) des feuilles, des tiges et de la plante entière de B. ruziziensis à la floraison.

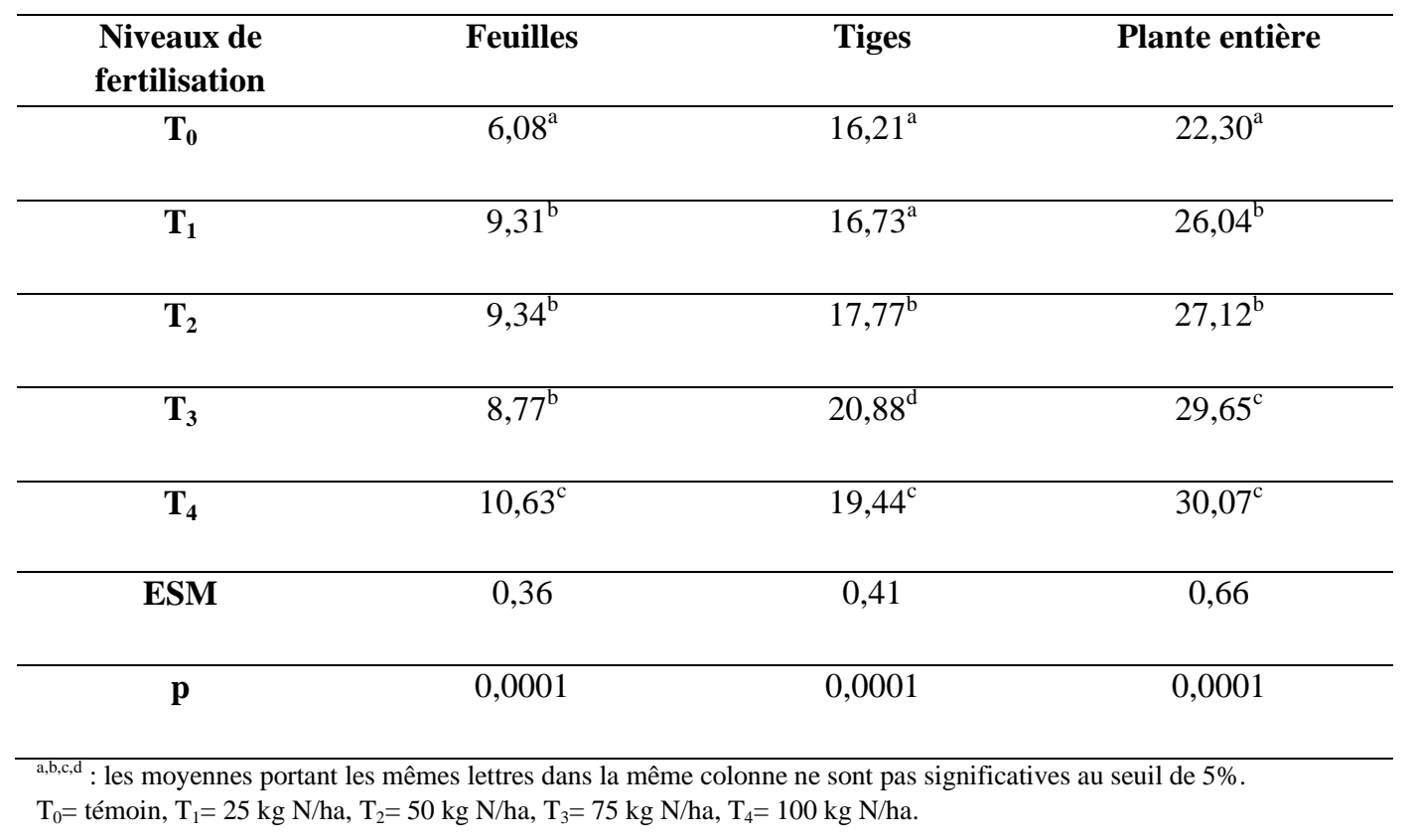

Tableau 7 : Effets des niveaux de fertilisation à la fiente de poules sur la production de biomasse (en t MS/ha) des feuilles, des tiges et de la plante entière de $B$. ruziziensis après grenaison.

\begin{tabular}{cccc}
\hline $\begin{array}{c}\text { Niveaux de } \\
\text { fertilisation }\end{array}$ & Feuilles & Tiges & Plante entière \\
\hline $\mathbf{T}_{\mathbf{0}}$ & $5,31^{\mathrm{a}}$ & $17,16^{\mathrm{a}}$ & $22,48^{\mathrm{a}}$ \\
\hline $\mathbf{T}_{\mathbf{1}}$ & $6,27^{\mathrm{b}}$ & $18,43^{\mathrm{b}}$ & $24,70^{\mathrm{b}}$ \\
\hline $\mathbf{T}_{\mathbf{2}}$ & $7,13^{\mathrm{c}}$ & $19,36^{\mathrm{bc}}$ & $26,49^{\mathrm{c}}$ \\
\hline $\mathbf{T}_{\mathbf{3}}$ & $7,24^{\mathrm{c}}$ & $19,76^{\mathrm{c}}$ & $27,00^{\mathrm{cd}}$ \\
\hline $\mathbf{T}_{\mathbf{4}}$ & $7,74^{\mathrm{c}}$ & $19,80^{\mathrm{c}}$ & 0,44 \\
\hline $\mathbf{E S M}$ & 0,21 & 0,260 & 0,0001 \\
\hline $\mathbf{p}$ & 0,0001 & 0,0001 & \\
\hline
\end{tabular}

a,b,c,d : les moyennes portant les mêmes lettres dans la même colonne ne sont pas significatives au seuil de $5 \%$.

$\mathrm{T}_{0}=$ témoin, $\mathrm{T}_{1}=25 \mathrm{~kg} \mathrm{~N} / \mathrm{ha}, \mathrm{T}_{2}=50 \mathrm{~kg} \mathrm{~N} / \mathrm{ha}, \mathrm{T}_{3}=75 \mathrm{~kg} \mathrm{~N} / \mathrm{ha}, \mathrm{T}_{4}=100 \mathrm{~kg} \mathrm{~N} / \mathrm{ha}$. 


\section{DISCUSSION}

Effet des niveaux de fertilisation à la fiente de poules sur la taille et le diamètre de $B$. ruziziensis à différents stades phénologiques

La fertilisation a permis d'obtenir les tailles et les diamètres des plantes des parcelles fertilisées supérieures à celles des plantes des parcelles non fertilisées. De plus, les tailles des plants de $B$. ruziziensis ont augmenté avec les niveaux de fertilisation aux fientes de poules à tous les stades phénologiques. La grande concentration en nutriments de la fiente de poules, spécialement en azote $(\mathrm{N})$ et en phosphore $(\mathrm{P})$ est à l'origine des améliorations dans la croissance de la plante (Yerima et al., 2014) observées à la montaison, à la floraison et après grenaison. Ceci a amené à penser que la synchronisation de la libération des éléments nutritifs par les fientes de poules pendant leur décomposition et leur assimilation par les plantes a été bonne. En effet, Mulaji (2011) et Kimuni et al. (2014) ont démontré que le taux de décomposition de la matière organique et la croissance des plantes était étroitement lié à la synchronisation entre la libération des nutriments et leur assimilation par la plante.

L'azote étant le principal facteur limitant la croissance et la production des plantes (Obulbiga et Kaboré-Zoungrana, 2007 ; Pamo et al., 2008 ; Tendonkeng et al., 2009), les différences observées entre les tailles des plantes des parcelles fertilisées et celles des plantes des parcelles non fertilisées peuvent s'expliquer par le fait que les faibles teneurs en azote $(0,6 \mathrm{~g} / \mathrm{kg}$ de sol) contenues dans le sol des parcelles non fertilisées ne correspondaient pas à la quantité nécessaire pour une croissance optimale de cette plante. Ainsi les apports d'azote sous forme de fiente de poules ont permis d'obtenir des différences significatives sur les tailles des plantes des parcelles fertilisées. Par ailleurs, les résultats des études menées par Yerima et al. (2014) ont montré que la fiente de poules améliore de façon significative les propriétés du sol, les rendant ainsi plus productifs. Cela met en exergue le rôle des fientes de poules sur la croissance et le développement des plantes.

Les résultats obtenus de l'effet des niveaux de fertilisation à la fiente de poules sur l'évolution de la taille et du diamètre de $B$. ruziziensis sont en concordance d'une part avec ceux obtenues par Tendonkeng en 2011. Résultats selon lesquels les tailles et les diamètres des plants de $B$. ruziziensis augmentaient avec les niveaux croissants de fertilisation azotée. D'autres parts avec ceux de Zamil et al. (2004) et Yerima et al. (2014) qui ont démontré que la fiente de poules améliore la disponibilité de l'azote, du phosphore et du potassium, ce qui favorise la croissance des plantes.

Effets des différents niveaux de fertilisation à la fiente de poules sur la production de biomasse de $B$. ruziziensis à différents stades phénologiques

La fertilisation à la fiente de poules a influencé positivement la production de biomasse des feuilles, des tiges et de la plante entière de $B$. ruziziensis à la montaison, floraison et après grenaison. Au niveau de la plante entière, chaque niveau supérieur de fertilisation a permis d'obtenir une biomasse significativement élevée par rapport aux niveaux de fertilisations inférieures. Les différences significatives observées entre les biomasses des différents niveaux de fertilisation s'expliquent, par le fait que les niveaux croissants de fertilisation à la fiente de poules ont fourni de façon proportionnelle aux quantités de fientes appliquées des éléments nutritifs nécessaires à la production de biomasse des plantes.

Les différences significatives observées entre les biomasses des différentes parties des plantes des parcelles fertilisées et celles des parcelles témoins s'expliquent par le fait que la fiente de poules est un fertilisant capable de redresser la balance cationique et le rapport $\mathrm{C} / \mathrm{N}$ du sol comme a démontré Yerima et al. (2014). D'une part, la matière organique apportée par la fiente de poule, et qui joue un rôle important dans le sol, va s'avérer favorable à la croissance des microorganismes qui vont induire une activation de la solubilisation des éléments nutritifs. Les éléments nutritifs rendus suffisamment disponibles au fil du temps dans le sol sont alors efficacement utilisés par les plantes cultivées (Ojetayo et al., 2011 ; Kimuni et al., 2014). C'est ainsi que la fiente de poules va apporter au sol des quantités suffisantes 
d'azote, de phosphore, de potassium, de calcium, et de magnésium et de matière organique (Agbede et al., 2008). Pendant la décomposition et la minéralisation de la matière organique, la libération des nutriments est bénéfique au sol de l'étude, pauvre en matière organique, azote, phosphore et en bases échangeables (Yerima et al., 2014). Ainsi, la matière organique apportée par la fiente de poule, va améliorer les propriétés physiques du sol. La densité apparente et la température du sol connaissent alors une réduction avec l'apport de matière organique (Agbede et al., 2008) tandis que la porosité totale, l'humidité et la capacité de rétention en eau sont améliorées. Cette dernière réduit la température journalière du sol réduisant ainsi la perte en eau (Akanni, 2005). La disponibilité croissante des nutriments du sol due à l'application de fumure augmente entrainant leur absorption par les racines des plantes (Yerima et al., 2014 ). Les résultats obtenus sur la fertilisation des plants de $B$. ruziziensis à base de fiente de poules corroborent avec celles des études précédentes réalisées par Agbede et al. (2008), Zamil et al. (2004) et Yerima et al. (2014) qui ont montré que la fiente de poules augmente la croissance et les rendements des plantes. Cela étant attribuable à l'amélioration des propriétés chimiques et physiques du sol, suite à cet apport des fientes de poules. Les biomasses des plantes entières des parcelles fertilisées à la fiente de poules à la dose de $100 \mathrm{~kg} \mathrm{~N} / \mathrm{ha}$ ont été supérieures à celles obtenues aux doses de fertilisation inférieures à tous les stades phénologiques. Ceci permet de dire que la fertilisation à la dose de $100 \mathrm{~kg} \mathrm{~N} / \mathrm{ha}$ était la fertilisation optimale pour la production de biomasse de $B$. ruziziensis.

\section{Conclusion}

Cette étude a été initiée en vue d'évaluer les effets des doses croissantes de la fiente de poules sur la croissance et la production de biomasse de Brachiaria ruziziensis. Ainsi, les tailles, les diamètres et les biomasses des différentes parties des plantes des parcelles fertilisées ont été supérieures à celui des parcelles témoins. Les augmentations observées dans les différents paramètres mesurés par rapport aux témoins ont été proportionnelles aux quantités de fientes répandues. La fertilisation à la dose de $100 \mathrm{~kg} \mathrm{~N} / \mathrm{ha}$ a donné les meilleurs rendements. En référence aux résultats obtenus, la fertilisation à la dose de $100 \mathrm{~kg}$ $\mathrm{N} / \mathrm{ha}$ conviendrait pour une culture optimale de $B$. ruziziensis afin de limiter les apports en engrais minéraux.

\section{CONFLIT D'INTÉRÊTS}

Les auteurs déclarent, sur l'honneur, qu'il n'existe aucun conflit d'intérêt ni entre eux, ni avec une structure ou organisation quelconque par rapport au présent article.

\section{CONTRIBUTIONS DES AUTEURS}

GJA, FT et DFW ont contribué à la mise en place du dispositif expérimental, la collecte et le traitement des données, la rédaction de l'article. VFN a contribué au traitement des données et la rédaction de l'article. ETP a supervisé toutes les activités de collecte des données et la rédaction de l'article.

\section{REMERCIEMENTS}

Les auteurs remercient l'Université de Dschang pour la mise à leur disposition du cadre approprié de recherche (Ferme d'Application et de Recherche ainsi que les Laboratoires de Production et de Nutrition Animales et de Botanique Appliquée).

\section{REFERENCES}

Agbede TM, Ojeniyi SO, Adeyemo AJ. 2008. Effect of poultry manure on soil physical and chemical properties, growth and grain yield of sorghum in southwest, Nigeria. American-Eurasian Journal Sustainable Agriculture, 2(1): 72-77.

Akanni DI. 2005. Response of nutrient composition and yield components of tomato livestock manure. Ph.D. Thesis, Department of Crop, Soil and Pest Management, Federal University of Technology, Akure. 120 p.

Cook BG, Pengelly BC, Brown SD, Donnelly JL, Eagles DA, Franco MA, Hanson J, Mullen BF, Partridge IJ, Peters M, Schultze-Kraft R. 2005. Tropical Forages: an interactive selection tool. 
[CD-ROM], CSIRO, DPI\&F(Qld), CIAT and ILRI, Brisbane, Australia.

Husson O, Charpentier H, Razanamparany C, Moussa N, Michellon R, Naudin K, Razafintsalama H, Rakotoarinivo C, Rakotondramanana, Seguy L. 2008. Brachiaria sp. Manuel pratique du semis direct à Madagascar. Volume III. $\begin{array}{lll}\text { Chapitre } & 4 . & \end{array}$ http://www.supagro.fr/ress-

pepites/PlantesdeCouverture/res/brachiar ia.pdf.

JGRC (Japan Green Resources Corporation). 2001. Guide technique de l'élevage: le développement pastoral efficace par la production d'herbe. In : Documentation technique de la Japan Green Resources Corporation: Générer l'abondance dans le sahel par la lutte contre la désertification. $68 \mathrm{p}$.

Kimuni NL, Mwali KM, Mulembo MT, Lwalaba LJ, Lubobo KA, Katombe NB, Mpundu MM, Baboy LL. 2014. Effets de doses croissantes des composts de fumiers de poules sur le rendement de chou de chine (Brassica chinensis L.) installé sur un sol acide de Lubumbashi. Journal of Applied Biosciences, 77: 6509-6522.

DOI: http://dx.doi.org/10.4314/jab.v77i1.4.

Mboko AV, Tendonkeng F, Matumuni FNE, Zougou GT, Miégoué E, Boukila B, Pamo TE. 2013. Effet comparé de deux légumineuses fertilisées au molybdène sur la croissance et le rendement de Brachiaria ruziziensis à différentes périodes de fauche dans l'ouest Cameroun. International Journal of Biological Chemical Sciences, 7(6): 2513-2525.

DOI: http://dx.doi.org/10.4314/ijbcs.7i6.26.

Mihoub A. 2008. Effet de la fertilisation phosphatée sur la nutrition azotée et la productivité d'une culture de blé dur (Triticum durum I. Var. carioca) dans la région d'El-Goléa-Ghardaia. Mémoire d'ingenierie en sciences agronomique, Université de Kasdi-Merbah Ouarla, Algérie.

http://www.mémoireonline.com/07/10/3 689/m_Effet-de-la-fertilisationphosphatee-sur-la-nutrition-azotee-et-la- productivite-dune-culture-de-6.html.

Muhindo KZ, Tendonkeng F, Miegoué E, Pamo TE. 2018. Influence de la fréquence de fauche sur la production de biomasse et la composition chimique du foin de Pennisetum clandestinum. International Journal of Biological and Chemical Sciences, 12(3):1459-1468. DOI: https//dx.doi.org/10.4314/ijbcs.v12i3.31.

Mulaji KC. 2011. Utilisation des composts de biodéchets ménagers pour l'amélioration de la fertilité des sols acides de la province de Kinshasa (République Démocratique du Congo). Thèse de doctorat, Université de Gembloux Agro bio tech, $220 \mathrm{p}$.

Obulbiga MF, Kaboré-Zoungrana CY. 2007. Influence de la fumure azotée et du rythme d'exploitation sur la production de matière sèche et la valeur alimentaire d'Andropogon gayanus kunth au Burkina Faso. Tropicultura, 25(3): 161167.

http://www.bib.fsagx.ac.be/tropicultura/t ext/v25n3.pdf.

Ojetayo AE, Olaniyi JO, Akanbi WB, Olabiyi TI. 2011. Effect of fertilizer types on nutritional quality of two cabbage varieties before and after storage. Journal of Applied Biosciences. 48: 3322- 3330 .

Pamo TE, Boukila B, Fonteh FA, Tendonkeng F, Kana JR, Nanda AS. 2007. Nutritive values of some basic grasses and leguminous tree foliage of the Central region of Africa. Animal Feed Science and Technology, 135: 273-282. DOI: http//doi.org/10.1016/J.anifeedsci.2006

Pamo TE, Boukila B, Fonteh FA, Tendonkeng F, Kana JR. 2005. Composition chimique et effet de la supplémentation avec Calliandra calothyrsus et Leucaena leucocephala sur la production laitière et la croissance des chevreaux nains de Guinée. Livestock Research for Rural Development, 17(3). Retrieved from http://www.cipav.org.co//rrd/lrrd17/03/te do17034.htm.

Pamo TE, Boukila B, Meduke CN, Tendonkeng F. 2008. Effect of nitrogen fertilisation and cutting frequency on the 
yield and regrowth of Panicum maximum Jacq. in West Cameroon. In: Xie Haining and Huang Jiehua (Eds). XXI International Grassland Congress / VIII International Rangeland Congress Hohhot, China, 29th June -5th July 2008. P. 354.

Pamo TE, Tendonkeng F, Kana JR, Boukila B, Nanda AS. 2006. Effect Calliandra calothyrsus and Leucaena leucocephala supplementary feeding goat production in Cameroon. Small Ruminant Research, 65: 31-37. DOI: 10.1016/j.smallrumres.2005.05.023

Sawa C, Tendonkeng F, Miegoue E, Beavogui F, Kourouma M, Pamo TE. 2018. Effet résiduel de la fertilisation minérale et du stade phénologique sur la croissance et la biomasse de Paspalum plicatum. International Journal of Biological and Chemical Sciences, 12(5): 2132-2142. DOI: https://dx.doi.org/10.4314/ijbcs.v12i5.15

Soltner D. 2003. Les bases de la Production Végétale. Tome 2: le sol et son amélioration. $18^{\mathrm{e}}$ Edition. Collection Sciences et Techniques Agricoles, $472 \mathrm{p}$. Tendonkeng F, Boukila B, Pamo TE, Mboko AV, Matumuini NEF, Fogang ZB. 2009. Effet de différents niveaux de fertilisation azotée et du stade phénologique sur la croissance et le rendement de Brachiaria ruziziensis dans l'Ouest Cameroun. International Journal of Biological and Chemical Sciences, 3(4): 725-735. DOI: 10.4314/ijbcs.v3i4.47182

Tendonkeng F, Boukila B, Pamo TE, Mboko AV, Matumuini NEF. 2011a. Effet direct et résiduel de différents niveaux de fertilisation azotée sur la croissance et le rendement de Brachiaria ruziziensis à différents stades phénologiques. Tropicultura, 29 (4): 197-204.
Tendonkeng F, Boukila B, Pamo TE, Mboko AV, Tchoumboué J. 2010. Effet de differents niveaux de fertilisation azotee sur le rendement et la composition chimique de Brachiaria ruziziensis à la montaison dans l'Ouest Cameroun. Livestock Research for Rural Development, 22: Article 19. Retrieved December 13, 2010, from http://www.Irrd.org/Irrd22/tend22019.ht $\mathrm{m}$.

Tendonkeng F, Boukila B, Pamo TE, Mboko AV, Zogang BF, Matumuini NEF. 2011b. Effets direct et résiduel de différents niveaux de fertilisation azotée sur la composition chimique de Brachiaria ruziziensis à la floraison à l'Ouest Cameroun. International Journal of Biological and Chemical Sciences, 5(2): 570-585.

Tendonkeng F. 2011. Effet de la fertilisation azotée, du stade phénologique et de l'année de fauche sur la croissance, le rendement et la valeur nutritive de Brachiaria ruziziensis Germain et Evrard. Thèse de Doctorat/Ph.D, Faculté d'Agronomie et des Sciences Agricoles. Université de Dschang Cameroun, 186 p.

Yerima BPK, Tiamgne AY, E Van Ranst. 2014. Réponse de deux variétés de tournesol (Helianthus sp.) à la fertilisation à base de fiente de poule sur un Hapli-Humic Ferrasol du Yongka Western Highlands Research Garden Park (YWHRGP) Nkwen-Bamenda, Cameroun, Afrique centrale. Tropicultura, 32(4):168-176.

Zamil SS, Quadir FQ, Chowdhury MAH, Al Wahid A. 2004. Effect of different animal manures on yield quality and nutrient uptake by mustard cv. Agrani. BRAC University Journal, 1(2): 56-66. 